\title{
Understanding and Avoiding AI Failures: A Practical Guide
}

\author{
Robert Williams * and Roman Yampolskiy (D) \\ Speed School of Engineering, University of Louisville, Louisville, KY 40292, USA; \\ roman.yampolskiy@louisville.edu \\ * Correspondence: robertmaxwilliams@gmail.com
}

Citation: Williams, R.; Yampolskiy, R. Understanding and Avoiding AI Failures: A Practical Guide. Philosophies 2021, 6, 53. https://doi.org/10.3390/ philosophies6030053

Academic Editor: Marcin J. Schroeder

Received: 7 May 2021

Accepted: 9 June 2021

Published: 28 June 2021

Publisher's Note: MDPI stays neutral with regard to jurisdictional claims in published maps and institutional affiliations.

Copyright: (c) 2020 by the authors. Licensee MDPI, Basel, Switzerland. This article is an open access article distributed under the terms and conditions of the Creative Commons Attribution (CC BY) license (https:// creativecommons.org/licenses/by/ $4.0 /)$.

\begin{abstract}
As AI technologies increase in capability and ubiquity, AI accidents are becoming more common. Based on normal accident theory, high reliability theory, and open systems theory, we create a framework for understanding the risks associated with AI applications. This framework is designed to direct attention to pertinent system properties without requiring unwieldy amounts of accuracy. In addition, we also use AI safety principles to quantify the unique risks of increased intelligence and human-like qualities in AI. Together, these two fields give a more complete picture of the risks of contemporary AI. By focusing on system properties near accidents instead of seeking a root cause of accidents, we identify where attention should be paid to safety for current generation AI systems.
\end{abstract}

Keywords: AI safety; normal accident theory; risk analysis

\section{Introduction}

With current AI technologies, harm done by AIs is limited to power that we put directly in their control. As said in Reference [1], "For Narrow AIs, safety failures are at the same level of importance as in general cybersecurity, but, for AGI, it is fundamentally different." Despite AGI (artificial general intelligence) still being well out of reach, the nature of AI catastrophes has already changed in the past two decades. Automated systems are now not only malfunctioning in isolation, they are interacting with humans and with each other in real time. This shift has made traditional systems analysis more difficult, as AI has more complexity and autonomy than software has before.

In response to this, we analyze how risks associated with complex control systems have been managed historically and the patterns in contemporary AI failures to what kinds of risks are created from the operation of any AI system. We present a framework for analyzing AI systems before they fail to understand how they change the risk landscape of the systems they are embedded in, based on conventional system analysis and open systems theory, as well as AI safety principles.

Finally, we present suggested measures that should be taken based on an AI system's properties. Several case studies from different domains are given as examples of how to use the framework and interpret its results.

\section{Related Work}

\subsection{Early History}

As computer control systems increased in complexity in the 1970s and 1980s, unexpected and sometimes catastrophic behavior would emerge from previously stable systems [2]. While linear control systems (for example, a thermostat) had been used for some time without unexpected behavior, adaptive control systems created novel and unexpected problems, such as "bursting". As described in Reference [2], bursting is the phenomenon where a stable controller would function as expected for a long time before bursting into oscillation, then returning to a stable state. This is caused by the adaptive controller not having a rich enough input during the stable period to determine the unknown 
coefficients of its model correctly, causing the coefficients to drift. Once the system enters oscillation, the signal again becomes rich enough for the controller to correctly estimate the unknown coefficients and the system becomes stable again. The increased complexity of the more advanced technology (dynamic controller instead of a static controller) introduced a dynamic not present in previous technologies, and incomprehensible to an operator not familiar with this behavior. Worse, since this behavior only happens when the controller is controlling the real world plant, designers had no way of predicting this failure mode. Bursting can be reduced using specifically engineered safety measures or more complex controllers (which bring even more confounding problems), but still demonstrates that increases in complexity tends to increase risk.

\subsection{Normal Accident Theory}

One of the principal values of 'normal accident' analysis and case descriptions is that it helps to develop convincing materials to counter the naive, perhaps wistful or shortsighted, views of decision-makers who, due to institutional pressure, desperation or arrogance, are tempted to make unrealistic assumptions about the systems they direct but for which they have only nominal operational responsibility. Todd R. La Porte [3]

Risk of failure is a property inherent to complex systems, and complex systems are inherently hazardous [4]. At a large enough scale, any system will produce "normal accidents". These are unavoidable accidents caused by a combination of complexity, coupling between components, and potential harm. A normal accident is different from more common component failure accidents in that the events and interactions leading to normal accident are not comprehensible to the operators of the system [5]. Increasing the complexity and broadening the role of AI components in a system decreases comprehensibility of the system, leading to an increase in normal accidents.

In 1984, Charles Perrow published "Normal Accidents" [5], which laid the groundwork for NAT (normal accident theory). Under NAT, any system that is tightly coupled and complexly interactive will inevitably experience a system accident. Decentralization reduces coupling and increases complexity, while centralization decreases complexity but also increases coupling. Thus, since an organization cannot be both centralized and decentralized, large organizations will harbor system properties that make them prone to normal accidents.

\subsection{High Reliability Theory}

High reliability theory was developed to explain the incredible ability of certain organizations to function without major accidents for long periods of time. In Reference [6], Weick identifies several common traits shared by high reliability organizations (HROs): a strategic prioritization of safety, careful attention to design and procedures, redundancy, decentralized decision-making, continuous training often through simulation, strong cultures that encourage vigilance and responsiveness to potential accidents, and a limited degree of trial-and-error learning.

High reliability organizations manage the apparent paradox proposed in normal accident theory by having traits of both centralization and decentralization. Decisionmaking is decentralized, allowing for decoupling, while policy and cultural factors are highly centralized, allowing for the unification of goals and attention to safety. This ability to be simultaneously centralized and decentralized through common culture and goals is present not only in HROs but in collectivist cultures, demonstrated by the tendency of members of these cultures to cooperate in social dilemmas $[7,8]$.

\subsection{NAT-HRT Reconciliation}

Normal accident theory holds that for industries with certain system properties, system accidents are inevitable. Meanwhile, high reliability theory makes the observation that there are many exceptions to this, and there are common traits shared by these HROs that can be studied and understood as indicators of reliability. In Reference [9], Shrivastava 
et al. analyze how the two theories appear to be in conflict then reconcile them by looking at how debates between the two sides neglect the importance of time in understanding accidents.

Normal accident theory states that a system has to choose to trade off between centralization (which allows for organization-aligned action and less chaos) and decentralization (which enables operators to quickly make decisions). High reliability theory considers that it is possible to escape this apparent paradox by allowing operators a high level of autonomy (decentralized decision-making) while also putting a focus on cultural aspects that improve safety and reliability (centralized goals).

Shrivastava et al. use the Swiss cheese model (SCM) to explain the importance of time in the occurrence of accidents, even in systems that are stable over time. In the SCM, the layers of safety are modeled as slices of cheese with holes in them representing weak points in each layer of safety. Over time, holes change shape and move around. Eventually, no matter how many slices of cheese (layers of safety) there are, the holes will align allowing a straight shot through all of the slices of cheese (an accident occurs) [10]. However, the SCM model only demonstrates that accidents are inevitable, and "inevitability is immaterial for practical purposes", as Shrivastava et al. state, since the time scales involved for some systems may be far longer than the system is expected to operate.

Disaster incubation theory (DIT) is introduced as the final piece needed to reconcile normal accident theory and high reliability theory. DIT describes how organizations gradually migrate to the boundary of acceptable behavior, as good safety records drive up complacency and deviance is normalized [11,12]. DIT was considered only useful in hindsight by proponents of NAT, so Snook [13] investigated accidents with this in mind and created the framing of "practical drift". This is the "slow steady uncoupling of local practice from written procedure" [13] which leads an initially highly coupled system to become uncoupled as operators and managers optimize their processes to be more efficient, deviating from procedure. Then, if the system is required to become tightly coupled again, the operators are ill-prepared for this increase in coupling and a system accident can occur.

Through the lens of disaster incubation theory and practical drift, Shrivastava et al. explain how NAT and HRT work to compliment each other to explain how accidents take place and are avoided. The time period being considered by HRT takes place while the system is still a high reliability organization. The culture and procedures put in place are working correctly, coupling is high, and complexity is manageable to the well trained operators. Over time, however, practical drift decouples the system and reliability decreases. If the organization is a high reliability organization, degradation is limited and incidents can still be managed. Accidents that take place during this period of time are within the scope of HRT. However, there is the possibility for an unlikely event to lead from this steady decline of reliability to a normal accident. If the system suddenly has to become more coupled (for instance, during a special mission or to react to an incident breaking down multiple layers of safety), it is ill prepared to do so. At this point, NAT's trade-off between coupling and complexity becomes important, and the perceived complexity of the system increases drastically, making safe operation impossible, leading to a normal accident. This can only happen after a great decrease in coupling from the initial (designed) state of the system, so proponents of HRT would say that the accident was only able to take place due to the system no longer acting as a high reliability organization.

High reliability theory explains how organizations resist practical drift, and the accidents that happen when practical drift leads to a breakdown of high reliability practices. Normal accident theory is useful when practical drift has lead to a great degree of decoupling, and then a sudden change in situation (which may be an intentional operation or an unexpected incident) requires increased coupling, which the system is (surprisingly, to operators) no longer able to handle without increasing complexity beyond manageable levels. 


\subsection{Lethal Autonomous Weaponry}

The introduction of lethal autonomous weaponry [14] increases the danger of normal accidents not because it provides new kinds of failure or novel technologies but because of the drastically increased potential harm. A machine which kills when functioning correctly is much more dangerous in an accident than one which only does harm when malfunctioning. By increasing the level of complexity and autonomy of weapons systems, normal accidents involving powerful weapons becomes a possibility.

\subsection{Robustness Issues for Learned Agents}

In Reference [15], Uesato et al. train a reinforcement learner in an environment with adversarial perturbations instead of random perturbations. Using adversarial perturbations, failure modes that would be extremely unlikely to be encountered otherwise were detected and integrated into training. This shows that AI trained to be "robust" by training in a noisy environment may still have catastrophic failure modes that are not observed during training, which can spontaneously occur after deployment in the real world. Adversarial training is a tool to uncover and improve these issues. However, it is only an engineered safety measure over the deeper issue of black box AI, which are not characterized of their entire input space.

Image classifiers famously fail when faced with images that have been modified by as little as one pixel [16], called adversarial examples. Despite performing well on training data, test data, and even data from other datasets, image classifiers can be made to reliably misclassify images by changing the images so slightly that the alterations are invisible to the casual observer. In Reference [17], Ilyas et al. argue that these misclassifications are not due to a simple vulnerability, but due to image classifiers' reliance on non-robust features. These are features which are not apparent to the human eye but can be used to accurately classify images, even those outside of the original dataset. Non-robust features are transferable to other datasets and the real world. However, they are also invisible to the human eye and can be altered without noticeably changing the appearance of the image. Classifiers with only robust features can be created through robust training [18], but they suffer from decreased accuracy. Thus, non-robust features are a useful mechanism to achieve high accuracy, at the cost of vulnerability to adversarial attacks.

The difference between robust and non-robust features is strictly human-centric. Ilyas et al. frame this as an alignment problem. While humans and image classifiers are superficially performing the same task, the image classifiers are doing it in a way the is incomprehensible to humans, and can fail in unexpected ways. The misalignment between the objective learned from the dataset and the human notion of an image belong to any particular class is the underlying cause for the effectiveness of adversarial examples.

This is a useful framing for other domains, as well. A reinforcement learner achieving impossibly high scores by hacking its environment does not "know" that it is breaking the rules-it is simply doing what was specified, and incredibly well. Just as a robust classifier loses some accuracy from being disallowed non-robust features, a reinforcement learner that is prevented from reward hacking will always obtain a lower reward. This is because while the designer's goal is to create a useful agent, the agent's goal is to maximize reward. These two are always misaligned, a problem referred to as the alignment problem [19].

\subsection{Examples of AI Failures}

Large collections of AI failures and systems to categorize them have been created before $[1,20]$. In Reference [20], the classification schema details failures by problem source (such as design flaws, misuse, equipment malfunction, etc.), consequences (physical, mental, emotional, financial, social, or cultural), scale of consequences (individual, corporation, or community), and agency (accidental, negligent, innocuous, or malicious). It also includes preventability and software development life-cycle stage.

The AI Failures Incident Database provides a publicly accessible view of AI failures [21]. There are 92 unique incidents the have been reported into it. A review of the 
agency (cause) of each one is not listed on the website, so we give an overview here. Of the 92 incidents, 8 have some degree of malicious intent. Three are cases where social media users or creators manipulated AI to show inappropriate content or cause bots to produce hate speech. Two are incidents of hacking: spoofing biometrics and stealing Etherium cryptocurrency. One is the use of AI generated video and audio to misrepresent a public official. The last two are the only cases where AI could be seen as a malicious agent. In one case, video game AI exploited a bug in the game to overpower human players. In another, bots created to edit Wikipedia competed in a proxy war making competing edits, expressing the competing desires of their human creators. If these examples are representative, then a majority of AI incidents happen by accident, while less than $10 \%$ are the result of malicious intent. The 2 examples of AI malicious intent intent can be ascribed to AI given goals which put them in opposition of others: countering edits in one case, and waging warfare (within a video game) in the other.

\subsection{Societal Impact of AI}

The proliferation of AI technologies has impacts in our socioeconomic systems and environment in complicated ways, both positive and negative [22]. AI has the ability to make life better for everyone but also to negatively impact many by displacing workers and increasing wealth disparity [23]. This is just one example of AI interacting with a complicated system (in this case, the job market) to have large scale consequences. While the effects of AI technologies on society and culture are outside of the scope of this paper, we expect that AI will continue to increase in ubiquity and with it the increased chance for large scale AI accidents.

\subsection{Engineered Interpretability Measures}

In Reference [10], Nushi et al. present Pandora, a state of the art image captioning system with novel interpretability features. It clusters its inputs and predicts modes of failure based on latent features. The image captioning system is broken down into three parts, the object detector, the language model, and the caption reranker. Pandora is able to predict error types based on the inputs to each of these models, as well as the interactions between small errors in each part of the system accumulating to a failure. This feature is of particular interest, as it is a step towards improved interpretability for complex systems. Pandora is remarkable for creating explanations for a black-box model (an artificial neural network) by training a trivially interpretable model (a decision tree) on the same inputs with failure modes as its output.

In Reference [24], Das et al. present an explanation generating module for a simulated household robot. A sequence-to-sequence model translates the robot's failure state to a natural language explanation of the failure and why it happened. Their results are promising - the explanations are generated reliably ( $90 \%$ accuracy), and the model only makes mistakes within closely related categories. The generated explanations improve the accuracy of an inexperienced user for correcting the failure, especially when the explanation contains the context of the failure. For example, instead of the robot stating only "Could not move its arm to the desired object.", it also gives a reason: "Could not move its arm to the desired object because the desired object is too far away."

Both of these papers offer major contributions to making AI components and systems comprised of them safer and more understandable. However, measurement mechanisms can create even greater degrees of confusion when they fail, and can delay or confound the diagnosis of a situation. When two pressure gauges were giving conflicting information at the Three Mile Island accident [5] (p. 25), they increased confusion instead of providing information. An AI which says nothing except that a malfunction has occurred is easier to fix than one that gives misleading information. If the accuracy is high enough, if the explanations are bounded for how incorrect they can be, or if the operator knows to secondguess the AI, then the risks created by an explanation system are limited. The utility of providing explanations of failures, as demonstrated in Reference [24], is a compelling 
reason to add interpretability components despite the additional complexity and unique risks they create. Further study into real world applications of these systems is needed to understand the pros and cons of implementing them.

\subsection{The AI Accident Network}

Attributing fault is difficult when AI does something illegal or harmful. Punishing the AI or putting it in jail would be meaningless, as our current level of AI lacks personhood. Even if trying an AI for crimes was deemed meaningful, traditional punishment mechanisms could fail in complex ways [25]. Instead, the blame must fall on some human or corporate actor. This is difficult because the number of parties responsible for the eventual deployment of the AI into the world could be large: the owner of the AI, the operator, the manufacturer, the designer, and so on. Lior [26] approaches this problem from a variety of legal perspectives and uses a network model of all involved agents to find the party liable for damages caused by an AI. A compelling anecdote introduces the problem: a child is injured by a security robot at a mall. Who is liable for the child's injuries? Lior frames liability from the paradigm of "nonreciprocal risk", which states that, "[if] the defendant has generated a 'disproportionate, excessive risk of harm, relative to the victim's risk-creating activity', she will be found liable under this approach."

Lior argues against other definitions of liability and that nonreciprocal risk is the best way to determine liability in the context of AI accidents. Lior then describes how the importance of different agents in an accident can be understood using network theory. By arranging the victims, AI, and responsible or related parties into a network, network theory heuristics can be used to locate parties liable for damages. These heuristics include the degree of a node (its number of connections), measures of centrality of a node in the network, and others.

As AI technologies are proliferated, AI accidents are happening not only in commercial settings (where damages are internal to the corporation and assigning blame is an internal matter) but in public settings, as well. Robots causing injuries to visitors and autonomous vehicles being involved in crashes are both novel examples of this. For companies planning on using AI in externally deployed products and services, understanding how AI liability is going to be treated legally is crucial to properly managing the financial and ethical risks of deployment.

\subsection{AI Safety}

The field of AI safety focuses on long-term risks associated with the creation of AGI (artificial general intelligence). The landmark paper "Concrete Problems in AI Safety" [27] identifies the following problems for current and future AI: avoiding negative side effects, avoiding reward hacking, scalable oversight, safe exploration, and robustness to distributional change. Other topics identified as requiring research priority by other authors include containment [28], reliability, error tolerance, value specification [29,30], and superintelligence [31,32]. These topics are all closely related and could all be considered an instance of the "Do What I Mean" directive [33]. We will explore the five topics from Reference [27] with examples of failures and preventative measures where applicable.

\subsubsection{Avoiding Negative Side Effects}

This problem has to do with things that are done by accident or indifference by the AI. A cleaning robot knocking over a vase is one example of this. Complex environments have so many kinds of "vases" that we are unlikely to be able to program in a penalty for all side effects [27]. A suite of simulated environments for testing AI safety, the AI Safety Gridworlds, includes a task of moving from one location to another without putting the environment in an irreversible state [34]. Safe agents which avoid side effects should prefer to avoid this irreversible state. To be able to avoid negative side effects, an agent has to understand the value of everything in its environment in relation to the importance of its objective, even things that the reward function is implicitly indifferent towards. Knocking 
over a vase is acceptable when trying to save someone's life, for example, but knocking over an inhabited building is not. Many ethical dilemmas encountered by people are concerned with weighing the importance of various side effects, such as environmental pollution from industrial activity and animal suffering from farming. This is a non-trivial problem even for humans [35]. However, using "common sense" to avoid damaging the environment while carrying out ordinary tasks is a realistic and practical goal with our current AI technologies. Inverse reward design, which treats the given reward function as incomplete, is able to avoid negative side effects to some degree [30], showing that making practical progress in this direction is achievable.

\subsubsection{Avoiding Reward Hacking}

Most AI systems designed today contain some form of reward function to be optimized. Unless designed with safety measures to prevent reward hacking, the AI can find ways to increase the reward signal without completing the objective. These might be benign, such as using a bug in the program to set the reward to an otherwise unattainably high value [36]; complicated, such as learning to fall over instead of learning to walk [37]; or dangerous, such as coercing human engineers to increase its reward signal by threatening violence or mindcrime [32].

Agents that wish to hack their rewards can do so by breaking out of their containers, whether they are within simple training environments or carefully engineered prisons with defenses in depth [28]. When researching a novel AI technology that has a risk of creating AGI, researchers must use safety measures to prevent potential AGI from escaping confinement. This is not common practice as AI today are not believed to be capable of escaping the simulations they are tested in. However, humans and AI have both broken confinement in games that are used to benchmark AI. In 2018, an AI designed to maximize the score in a video game instead performed a series of seemingly random actions which caused the score to increase rapidly without continuing to progress in the game [36]. In this example, the $\mathrm{AI}$ is supposed to be using the game controller output to move the character around the screen to play the game and maximize the score, but it instead learned to hack the game and obtain a much higher score than is conventionally possible. Through an exploit in the game's programming the AI managed to directly modify its score instead of taking actions within the simulation as expected. In 2014, an exploit in Super Mario World was used to modify memory and jump directly to the end screen of the game a few minutes into play. This also demonstrates an agent (in this case, a computer assisted human) breaking a level of containment through a sophisticated attack.

In light of these examples, it is possible to gain arbitrary code execution abilities from within a sandboxed game environment and these kinds of exploits may be present inside any program which is meant to limit the output space of an AI. Virtual machines, often used as an additional layer of security, are also susceptible to a wide range of exploits [38]. This illustrates a more general concern which is the AI acting outside of the output space that it was designed to work with, seen in many of the AI failures in Reference [1].

Adversarial reward functions, where the reward function is learned and has a competing objective with the AI, can decrease reward hacking [39]. Another mitigation strategy is the use of tripwires. For instance, if the AI gets a much higher score than expected, it can be assumed that it has broken containment and is modifying the reward directly. This can be used as a tripwire which, when triggered, deactivates the AI. In Reference [40], Carlson describes how distributed ledger technology ("blockchain") can be used to create tripwires and other AI safety measures that cannot be corrupted by conventional means.

\subsubsection{Scalable Oversight}

Designers only have limited resources to monitor the actions of the AI. External factors are inevitably forgotten about (worsening side effects) and reward functions fail to perfectly capture what we really want (worsening reward hacking). We cannot monitor every action taken by an AI at every moment because they operate too fast or in a way that's too complex 
for us to understand, and because the goal is often to automate a task specifically so it can be completed without constant human oversight. Semi-supervised learning [27] is a first step towards scalable oversight as it allows labeled and unlabeled data to be used to train an AI. In an online learning context, this means that the AI can learn by doing the task while only occasionally needing feedback from a human expert. Semi-supervised learning is useful in terms of data efficiency and is a promising avenue for creating scalable oversight for AGI.

When dealing with AGI, scalable oversight is no longer an issue of data efficiency or human effort but of safety. At some point, the AGI will be intelligent enough that we are not able to tell if it is acting in our best interest or deceiving us while actually doing something unsafe. Scalable oversight is required to make AGI safe. One phrasing of a scalable oversight from the perspective of the AGI is "If the designers understood what I was doing, they would approve of it."

\subsubsection{Safe Exploration}

Reinforcement learning algorithms, such as epsilon-greedy and Q-learning, occasionally take a random action as a way to explore their environments. This is effective, especially with a small environment and a very large amount of training time, but it is not safe for real world environments. You do not want your robot to try driving over a cliff for the sake of exploration. Curiosity-driven exploration is able to efficiently explore video game environments by using an intrinsic reward function based on novelty [41]. This provides great benefits to the learner, but cannot ensure safety. For example, the agent only learns to avoid death because restarting the level is "boring" to it. A backup policy which can take over when the agent is outside of safe operating conditions can allow for safe and bounded exploration, such as an AI controlled helicopter which is switched to a hover policy when it gets too close to the ground or travels too fast [42].

Robotics controlled by current narrow AI need to be designed to avoid damaging expensive hardware or hurting people. Safe exploration becomes more difficult with increased intelligence. A superintelligent AGI might kill or torture humans to understand us better or destroy the Earth simply because such a thing has never been done before.

\subsubsection{Robustness to Distributional Change}

AI that has been trained in one environment or dataset may fail when its use is greatly different from its training. This is the cause AI obtaining good accuracy on curated datasets but failing when put into the real world. Distributional shift can also be seen in the form of racial bias of some AI [43].

\subsection{The Interpretation Problem}

The difficulty of converting what we want into rules that can be carried out by an AI has been named the "interpretation problem" [44]. This problem arises in domains outside of AI, such as sports and tax law. For sports, the rules of a game are written to reflect a best effort representation of the spirit of the game to bring out creativity and skill in the players. The players, however, seek only to win and will sometimes create legal tactics which "break" the game. At this point, to keep the game from stagnating, the rules are modified to prohibit the novel game-breaking strategy. A similar situation takes place in tax law, with friction between lawmakers and taxpayers creating increasingly complex laws.

In AI safety, the designers knows what is and is not morally acceptable from their point of view. They then design rules for the AI to abide by while seeking its objective and craft the objective to be morally acceptable. The AI, however, only has access to the rules and not to the human values that created them. Because of this, it will misinterpret the rules as it pursues its objective, causing undesirable behavior (such as the destruction of humanity). Humans do not learn morality from a list of rules, but from some combination of innate knowledge and lived experience. Badea et al. suggest that the same applies to AI. Any attempt to create a set of rules to constrain the AI to moral behavior will fail due 
to the interpretation problem. Instead, we need to figure out how we can show the AI human moral values indirectly, instead of having to state them explicitly. Since our goals and "common sense" as applied to any real world environment are too complex to write down without falling prey to the interpretation problem, an alternative approach where the $\mathrm{AI}$ is able to acquire values from us indirectly is required instead. Badea et al. do not provide details on how this could be accomplished, but there are some technologies which are very similar in nature to the proposed value learning system. Inverse reinforcement learning [45] is able to learn objectives from human demonstrations, and can learn goals that would be difficult to explicitly state. Future developments in this area will be needed to create moral AI.

\section{Classification Schema for AI Systems}

To better understand an AI system and the risks that it creates, we have created a classification schema for AI systems. We present a tag-based schema similar to the one presented in Reference [20]. Instead of focusing on AI failures in isolation, this schema includes both the AI and the system the AI is embedded in, allowing for detailed risk analysis prior to failure. We pay particular attention to the orientation of the AI as both a system with its own inherent dangers and as a component in a larger system which depends on the AI. Any analysis attempting to divide a system into components must acknowledge the "ambiguities, since once could argue interminably over the dividing line between part, unit, and subsystem" [5]. The particular choice of where to draw the line between the AI, the system, and the environment are important to making a meaningful analysis, and requires some intuition. Because of this, our classification system encourages repeating parts of it for different choices of dividing lines between the AI and the system to encourage finding the most relevant framing.

To characterize the risk of an AI system, one factor is the dangers expressed by the larger system in which the AI is a component. In an experimental setting, a genetic algorithm hacking the simulator can be an amusing bug [37], but a similar bug making its way into an autonomous vehicle or industrial control system would be dangerous. Understanding the risks involved in operating the system that the AI belongs to is critical in understanding the risk from an AI application.

The output of an AI must be connected to some means of control to be useful within the system. This can take many forms: indirectly, with AI informing humans who then make decisions; directly with an AI controlling the actuators of a robot or chemical plant; or though information systems, such as a social media bot that responds to users in real time. Any production AI system has some degree of control over the world, and it may not be clear where the effects of the AI take place. The connection between the output of a component (the AI) and other components is an instance of coupling. Because there are multiple components affected by the AI and those components are themselves coupled with still other components, we frame this problem in terms of the AI, which has its own properties, and a series of targets which the AI can affect. For example, the output from an AI in a chemical plant can be framed in numerous ways: the software signal with the computer the $\mathrm{AI}$ is running in, the electrical output from the controller, the actuation of valve, the rate of fluid flowing through the valve, or a variable in the chemical reaction taking place downstream. Identifying the target of the AI requires context, and all of these targets have unique consequences the might be overlooked in analyzing just one of them. The classification schema allows for as many targets as needed when analyzing a system.

Observability of the warning signs of an accident is important as it allows for interventions by human operators, a crucial tool in preventing accidents ("People continuously create safety" [4].). The likelihood of timely human interventions depends on four things:

- $\quad$ Time delays between AI outputs and the effects of the target.

- $\quad$ Observability of the system state as it is relevant to potential accidents.

- $\quad$ Frequency and depth of attention paid by human operators.

- Ability of operators to correct the problem once it has been identified. 
The time delay between an AI creating an output and that output affecting the target is essential to preventing accidents. Tightly coupled systems with short time delays (such as automated stock trading) are more hazardous because the system can go from apparent normalcy to catastrophe faster than operators can realize that there is something wrong [46]. Observability and attention from human operators are needed for these time delays to be an effective component of safety. As the level of automation of a system increases, human operators become less attentive and their understanding of its behavior decreases [47]. Reliance on automated systems decreases an operator's ability to regain control over a system if an accident requires manual control. For example, if an autonomous driving system fails, the driver, now less familiar with driving, has to suddenly be in manual control. Together, observability, human attention, and human ability to correct possible failures in the system all make up a major factor in whether or not a malfunction leads to an accident.

For a given choice of target being controlled by the AI, there is maximum conceivable amount of damage that can be done by malicious use of that target. We use the figure as a cap as to the amount of harm possible. Most AI failures are not malicious (see the discussion on AI Incidents in Section 2.7), so the harm done by an accident will almost always be much less than this amount. This factor is very difficult to predict prior to an accident. For example, imagine a component which controls cooling for a nuclear plant. One could say the maximum damage from its malfunctioning is a lost day of productivity because the backup cooling system will prevent any permanent damage. Another might say loss of the entire core is possible if the backup system also malfunctions, but the containment system is sure to keep it from harming anyone outside. Yet another might notice that containment could also fail, and a meltdown could harm millions of people in the surrounding area. Because of the unpredictability of how much harm one system could do, this factor is included as a rough estimate of the scale of power the AI has over the system and the system has over the environment.

The final criteria for classifying the nature and degree of systems risk of an AI system are coupling, complexity of interaction, energy level, and knowledge gap. Coupling is a measure of the AI's interconnectivity with other components in the system, and the strength of these connections. Loosely coupled systems have sparse connectivity which limits the propagations of component failure into an accident, but are also less robust. Tightly coupled systems have dense connectivity and many paths between components, and often feedback loops that allow a component to affect itself in complicated ways [5]. Classifying the level of coupling of the system in proximity to the AI component can be difficult and nebulous, so only course categories (loose, medium, tight) are used in this analysis as finer grained considerations are likely to become arbitrary.

Coupling considers other components and aspects of the environment that the AI is coupled with. Examples of coupling include taking data in from another component, transmitting data to another component, relying on the functioning of a component, and having another component rely on the functioning of the AI. The AI component is scored on a scale of 1-5 from loosely coupled to strongly coupled.

Complexity of interaction is a measure of how complex these interactions are. Linear interactions are simple to understand and behavior is easy to extrapolate from a few observations. Complex interactions have sudden non-linearities and bifurcations. The difference is ultimately subjective, as what appears to be a complex process to an inexperienced operator can become linear with experience and time [5]. An estimate of complexity of the AI's interactions within the system is made on a scale of 1-5.

Energy level is the amount of energy available to or produced by the system. Low energy systems are systems such are battery operated devices, household appliances, and computers. Medium energy systems include transportation systems, small factories, and mining. High energy systems include space travel, nuclear power, and some chemical plants [5]. 
Knowledge gap is the gap between how well technology is understood and the scale of its use. Technologies very early in the technological readiness scale [48] have a large knowledge gap if used in production systems. Likewise, technologies that are very well developed and understood can be used in production without creating a knowledge gap, so long as appropriately trained personnel are operating them. The tendency to have fewer and less catastrophic failures as a technology matures is due to the decreased knowledge gap as understanding increases. Energy level and knowledge gap together can be plotted along two axis to estimate the scale and degree of catastrophic risk, shown in Figure 1.

Analysis done in the field of AI safety frequently addresses dangers associated with artificial general intelligence (AGI) and superhuman intelligence. In Section 1, we gave an cursory overview of the dangers of AGI. Certain classes of contemporary AI failures are closely related to those that true AGI could manifest, indicating that considerations, such as containment and reward hacking, are useful in analyzing AI applications, even if the existential threat of AGI is not present. We also include a risk factor for research which may lead to the development of AGI in our analysis. These dangers are treated from an AI safety perspective as opposed to conventional safety considerations.

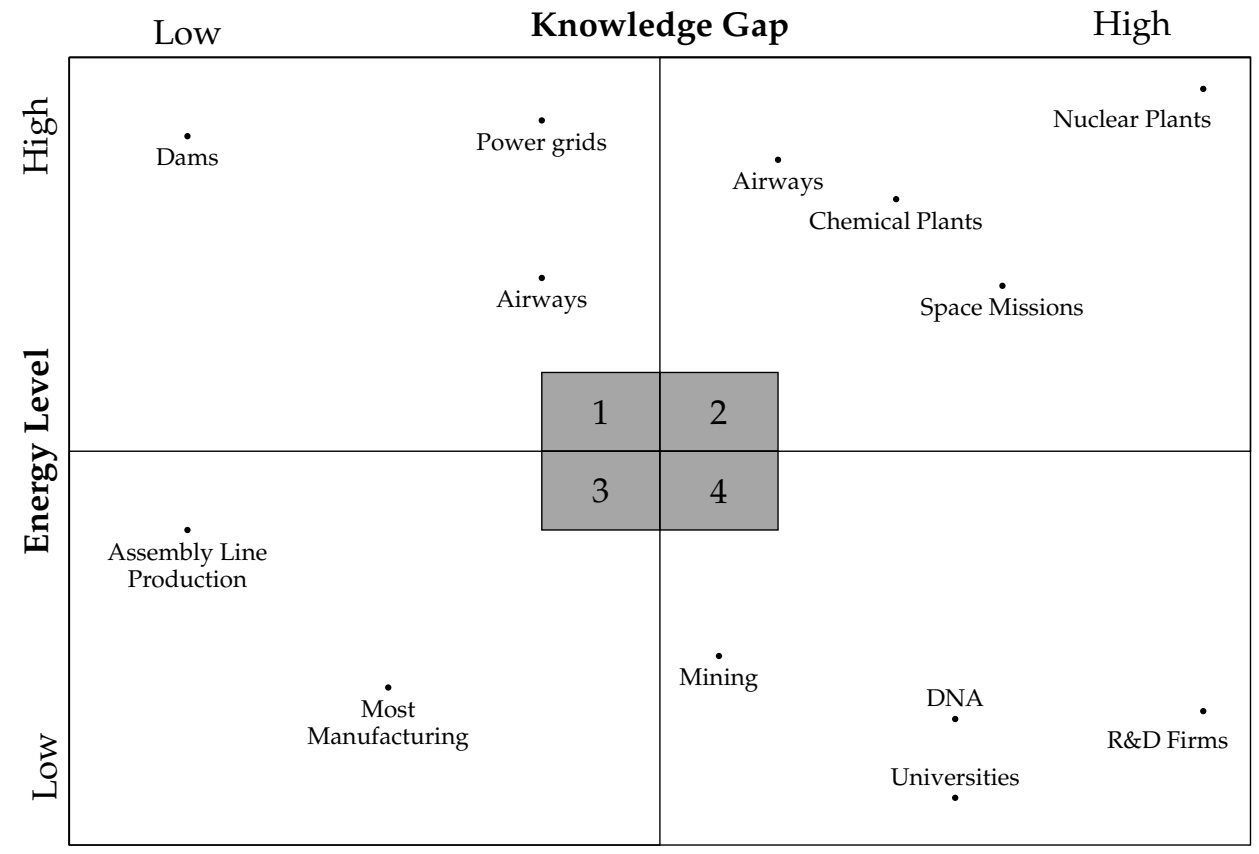

Figure 1. Plotting energy level against knowledge gap to create 4 quadrants. Systems in quadrant 3 have the lowest damage potential, and damage is limited to 1st parties. Systems in quadrant 4 have moderate damage potential up to 4 th party victims, systems in quadrant 1 have high damage potential up to third party victims, and systems in quadrant 1 have catastrophic risk potentials to 4 th party victims. Based on Shrivastava et al. [9] (p. 1361).

The following factors are considered most significant to understanding the level and nature of the risk of a system utilizing AI:

- $\quad$ The system which is affected by the outputs of the AI.

- Time delay between AI outputs and the larger system, system observability, level of human attention, and ability of operators to correct for malfunctioning of the AI.

- The maximum damage possible by malicious use of the systems the AI controls.

- Coupling of the components in proximity to the AI and complexity of interactions.

- Knowledge gap of AI and other technologies used and the energy level of the system.

\subsection{Timely Intervention Indicators}

Time delay, observability, human attention, and correctability make of the four factors for determining the ability of human operators to make a timely intervention in the case 
of an accident. These indicators are shown in Table 1. Time delay is how long it takes for the AI component to have a significant effect on the target in question. Only an order of magnitude ("minutes" vs. "hours" vs. "days") is needed.

Observability measures how observable the internal state of the system is, how often and with what degree of attention a human will attend to the system, and how easy or difficult a failure of the AI component of the system is to correct once detected. Observability is measured on a scale of $0-5$, from 0 for a complete black box to 5 for AI whose relevant inner workings can be understood trivially.

Table 1. Reference table for timely intervention indicators: time delay, observability, human attention, and correctability measures.

\begin{tabular}{cc}
\hline Timely & Possible Values \\
Intervention & \\
Indicators & (seconds, minutes, hours, etc.) \\
\hline Time Delay & $0-5$ \\
Observability & $($ N times per day) OR (intermittent, (days, weeks, months $)))$ \\
Human Attention & $0-5$ \\
Correctability & \\
\hline
\end{tabular}

Human attention is measured as the number of hours in a day that an operator will spend monitoring or investigating the AI component when there have not been any signs of malfunction. If the system is not monitored regularly, then this is instead written as the amount of time that will pass between checkups.

Correctability is the ability of an operator to manage an incident. If the AI can be turned off without disrupting the system or if a backup system can take control quickly, then correctability is high. Correctability is low when there is no alternative to the AI and its operation is necessary to the continued functioning of the system. Correctability is measured on a scale of $0-5$, from impossible to correct to trivially correctable.

\subsection{Target of AI Control}

All steps with an asterisk $\left(^{*}\right)$ should be repeated for each possible target. Targets should be chosen from a wide variety of scales to achieve the best quality of analysis. The data for each target should be listed as shown in Table 2 .

Table 2. Reference table for system risk factors for each target of AI control.

\begin{tabular}{cccc}
\hline Targets & Max Damage & System Accident Risk & Potential Damage to Other Parties \\
\hline Target 1 & $\$ \mathrm{x}$ & $(\mathrm{L}, \mathrm{M}, \mathrm{H})$ & $(\mathrm{L}, \mathrm{M}, \mathrm{H}, \mathrm{C})(1,2,3,4)$ \\
Target 2 & $\$ \mathrm{x}$ & $(\mathrm{L}, \mathrm{M}, \mathrm{H})$ & $(\mathrm{L}, \mathrm{M}, \mathrm{H}, \mathrm{C})(1,2,3,4)$ \\
$\ldots$ & & $(\mathrm{L}, \mathrm{M}, \mathrm{H})$ & $(\mathrm{L}, \mathrm{M}, \mathrm{H}, \mathrm{C})(1,2,3,4)$ \\
Target $\mathrm{n}$ & $\$ \mathrm{x}$ & & \\
\hline
\end{tabular}

\subsection{Single Component Maximum Possible Damage *}

This is the amount of damage that could be done by a worst case malfunctioning of the AI component in isolation. Since the actual worst case would be unimaginably unlikely or require superhuman AI in control of the AI component, we instead approximate the expected worst case by imagining a human adversary gaining control of the AI component and attempting to do as much harm as possible. This should consider both monetary damage, harm to people, and any other kinds of harm that could come about in this situation. 


\subsection{Coupling and Complexity *}

Together, coupling and complexity are used to asses the risk of experiencing a systems accident. Use Table 3 to convert coupling (high, medium, low) and interaction complexity (linear, moderate, complex) to find the risk of a systems accident (' $\mathrm{L}^{\prime},{ }^{\prime} \mathrm{M}^{\prime}$, ' $\mathrm{H}^{\prime}$, and ' $\mathrm{C}$ ' for low, medium, high, and catastrophic, respectively).

Table 3. Using coupling and interaction level to determine the severity of accident the system may experience. Adapted from Reference [9].

\begin{tabular}{cccc}
\hline \multirow{2}{*}{ Coupling } & \multicolumn{3}{c}{ Interaction } \\
\cline { 2 - 4 } & Linear & Moderate & Complex \\
\hline High & $\mathrm{M}$ & $\mathrm{H}$ & $\mathrm{C}$ \\
Medium & $\mathrm{L}$ & $\mathrm{M}$ & $\mathrm{H}$ \\
Low & $\mathrm{L}$ & $\mathrm{L}$ & $\mathrm{M}$ \\
\hline
\end{tabular}

\subsection{Energy Level and Knowledge Gap *}

Energy level and knowledge gap are used together to predict the potential damage from an accident and the degree of separation from the accident to potential victims using Table 4. The first letter is the amount of damage (' $\mathrm{L}$ ', ' $\mathrm{M}$ ', and ' $\mathrm{H}$ ' for low, medium, and high, respectively), and the number is the degree of separation between the system and the potential victims of the accident. First-party victims are operators, second-party victims are non-operating personnel and system users, third party victims are unrelated bystanders, and fourth party victims are people in future generations [9].

Table 4. Using energy level and knowledge gap to determine amount of damage (Low, Medium, High, Catastrophic) and distance to potential victims (1st party, 2nd party, 3rd party, 4th party). Adapted from Reference [9].

\begin{tabular}{cccc}
\hline \multirow{2}{*}{ Energy Level } & \multicolumn{3}{c}{ Knowledge Gap } \\
\cline { 2 - 4 } & Low & Med & High \\
\hline High & H3 & H3 & C4 \\
Med & M3 & M3 & H4 \\
Low & L2 & L2 & M4 \\
\hline
\end{tabular}

\section{AI Safety Evaluation}

In this section, we evaluate AI safety concerns, without being concerned about the details of the system the AI is being used in. While no current AI systems pose an existential threat, the possibility of a "foom" (The word "foom" denotes a rapid exponential increase of a single entity's intelligence, possibly through recursive self improvement, once a certain threshold of intelligence has been reached. It is also called a "hard takeoff" [49]). A "foom" scenario [31] is considered seriously as it is unknown how far we are from this point. Recent advancements (such at GPT-3 [50]) remind us how rapidly AI capabilities can increase. In this section we create a schema for classifying the AI itself in terms of autonomy, goal complexity, escape potential, and anthropomorphization.

\subsection{Autonomy}

We present a compressed version of the autonomy scale presented in Reference [51]. Autonomy is as difficult to define as intelligence, but the categories and examples given in Table 5 allow for simple, although coarse, classification. 
Table 5. AI autonomy levels with examples. Reinforcement learning agents and GA are considered highly autonomous due to their superhuman performance in Atari games [52] and robotic control [53]. The properties that allow for this performance also cause them to have high scores in the AI risk factors explained in this section.

\begin{tabular}{ccc}
\hline Autonomy Level & Description & Examples \\
\hline Level 0 & Little or no autonomy & PID controller, if-statement \\
\hline Level 1 & $\begin{array}{c}\text { Limited, well understood } \\
\text { optimization process }\end{array}$ & image classifier, GPT-2 \\
\hline Level 2 & $\begin{array}{c}\text { Agent with goals and iterated } \\
\text { interactions with an environment }\end{array}$ & $\begin{array}{c}\text { reinforcement learners, GA } \\
\text { learned agents }\end{array}$ \\
\hline Level 3 (danger) & $\begin{array}{c}\text { Agent capable of acting } \\
\text { independently on intrinsic } \\
\text { motivation }\end{array}$ & humans, AGI \\
\hline
\end{tabular}

On level 0, the AI (or non-AI program) has little or no autonomy. The program is explicitly designed and will not act in unexpected ways except due to software bugs. At level 1, an optimization process is used, but not one that has the capability of breaking confinement. Most AI today is at this level, where it is able to perform complex tasks but lacks any volition to do anything but transform inputs into outputs. Level 2 is closely related to level 1, except that the addition of iterated interactions with an environment allows for a greater degree of freedom and decision-making. Reinforcement learners and game-playing $\mathrm{AI}$ are in level 2, as the feedback loop of interacting with the environment creates an embodiment more similar to that of humans in our world. At level 3, the AI can be considered an AGI, as this level of autonomy requires a high degree of intelligence. It can understand the agency of others in the environment (theory of other minds) and seek to sustain and improve itself, and seek to escape confinement.

\subsection{Goal Complexity}

Software that has very simple functionality (such as a thermostat) has completely comprehensible goals, to the extent that we hardly consider them goals so much as the system's only functionality. More complex software may use an optimization process to maximize some objective function (or minimize a loss function). This provides a goal which is understandable but the goal seeking behavior of the agent may not be. Goal complexity levels are detailed in Table 6.

Table 6. AI goal complexity with examples.

\begin{tabular}{ccc}
\hline $\begin{array}{c}\text { Goal Complexity } \\
\text { Level }\end{array}$ & Description & Examples \\
\hline Level 0 & $\begin{array}{c}\text { Programs that "just run" and have } \\
\text { no goal seeking behavior }\end{array}$ & conventional software \\
\hline Level 1 & Basic goal seeking behavior & numerical optimization \\
\hline Level 2 & $\begin{array}{c}\text { Complex goal seeking with the } \\
\text { ability to seek subgoals and express } \\
\text { reward hacking }\end{array}$ & $\begin{array}{c}\text { genetic algorithm, } \\
\text { reinforcement learner }\end{array}$ \\
\hline Level 3 (danger) & $\begin{array}{c}\text { Ability to realize instrumental goals } \\
\text { and seek self improvement, ability } \\
\text { to hide goals }\end{array}$ & humans, AGI \\
\hline & & \\
\hline
\end{tabular}

On level 0 , the program does not exhibit any goal directed behavior. This is how we typically expect software to act-it just does what it is programmed to do. This is not to 
say that software always does exactly what it is programmed to do, as programmers make mistakes, but that programs of this sort lack goals. At levels 1 and 2, the program has been given an objective and will search for novel ways to reach the objectives, often creating surprising results to the programmers. The actual goal is known, but the realization of that goal and the implicit creation of subgoals leads to an increased difficulty in understanding what it is that the program is doing. At level 3, the program is able not only to formulate subgoals but also have instrumental goals and the ability to intentionally obfuscate its goals. This is seen in humans, who often obscure their motives, and in hypothetical AGI which deceive humans into thinking their goals are aligned with ours.

\subsection{Escape Potential}

AI confinement is a difficult problem with many proposed measures to improve security but no guarantees about safety $[28,54]$. However, our current AI either make no attempt to escape or lack the ability to break out of simulations. There are some examples of real world AI breaking a layer of confinement (see Section 2.11.2), but none that present any risk to humans or the capability establishing a persistent presence on remote computers. Escape potential levels are shown in Table 7.

Table 7. AI escape potential with examples.

\begin{tabular}{ccc}
\hline Escape Potential & Description & Examples \\
\hline Level 0 & None & conventional software \\
\hline Level 1 & $\begin{array}{c}\text { The agent may find unexpected } \\
\text { behaviors which technically achieve } \\
\text { the goal but are not desirable }\end{array}$ & genetic algorithms \\
\hline Level 2 & $\begin{array}{c}\text { The agent intentionally seeks to } \\
\text { hack its environment }\end{array}$ & $\begin{array}{c}\text { advanced reinforcement } \\
\text { learners }\end{array}$ \\
\hline Level 3 (danger) & $\begin{array}{c}\text { The agent has the ability and drive } \\
\text { to break through most cybersecurity } \\
\text { and containment procedures }\end{array}$ & AGI \\
\hline
\end{tabular}

At level 0, no confinement is needed beyond standard cybersecurity measures appropriate for the deployment environment. At levels 1 and 2, there is little risk of hostile escape but the agent should be monitored closely for undesirable behavior and software exploits. The distributional shift from the simulator to the real world provides a barrier against narrow AI affecting the real world. Because of this, agents trained in more realistic simulators should be regarded with a greater degree of caution. At level 3, indefinite confinement is impossible, but, with robust security, at the level of software, hardware, and social engineering, humans can benefit from a contained AGI in a limited capacity.

\subsection{Anthropomorphization}

The proliferation of humanoid, "friendly" AI products increases the degree to which people are willing to anthropomorphize software. This leads to misconceptions about approaches which are needed to manage existential risk and changes how researchers conceive of AI research and AI safety research [55].

Creating human-like AI has long been a goal for some researchers, as we expect AI which is able to emulate human behavior is capable of general intelligence. The Turing test was devised as a substitute for the question "do humans think?", based on the premise that imitating humans is a problem that can only be solved by a machine with human level intelligence. If a machine can reliably convince a human judge that it is human in a text-only conversation, then it passes the Turing test, and this suggests a high level of intelligence and possibly personhood [56]. Because of this, we consider an AI with level 3 anthropomorphization to be AGI. However, passing the Turing test does not imply 
that the AI has other human-like traits. Assuming that an AI is safe because it can pass the Turing test is dangerous because it does not necessarily have a similar architecture to a human. Table 8 shows four different levels of anthropomorphization for an AI and examples for each.

Table 8. AI anthropomorphization levels with examples.

\begin{tabular}{ccc}
\hline $\begin{array}{c}\text { Anthropomorphization } \\
\text { Level }\end{array}$ & Description & Examples \\
\hline Level 0 & No anthropomorphization & $\begin{array}{c}\text { home computer, a } \\
\text { calculator }\end{array}$ \\
Level 1 & $\begin{array}{c}\text { Some surface level appearance of } \\
\text { humanity (natural language } \\
\text { interaction, robot chassis with } \\
\text { human features, a name and } \\
\text { supposed personality) }\end{array}$ & ASIMO, robotic pets \\
\hline Level 2 & $\begin{array}{c}\text { Level 1 but with software to } \\
\text { support the illusion of humanity } \\
\text { (speech interaction, human-like } \\
\text { actuated movements) }\end{array}$ & Alexa, Sophia \\
\hline Level 3 (danger) & $\begin{array}{c}\text { The AI can be mistaken for a } \\
\text { human even with unrestricted } \\
\text { communication (may be text-only, } \\
\text { voice, or in person) }\end{array}$ & AGI \\
\hline
\end{tabular}

Levels 0 and 1 have been possible for a long time with minimal risks. However, the popularization of level 2 AI has created a social climate which harbors misunderstanding of the state of the art in AI and AI safety. While we are close to creating chatbots that can win at the restricted Turing test, these chat bots rely on psychological tricks to keep short-duration conversations within pre-constructed domains [57]. The creation of level 3 AI constitutes a dissolution of the boundaries between humans and AI and will likely require the creation of AGI.

\section{Determining Risk Using Schema Tags}

The following rules use schema tags developed in Section 3 to determine concrete steps needed for managing the risk of deploying an AI application.

Part of the danger of tightly coupled systems is the fast communication between nodes. On an assembly line there are at least a few seconds between one part of the process and another, and an operator is able to see the effects of an incident, intervening to prevent it from becoming an accident. In real time stock trading and nuclear power plant operation, there is neither the time nor the observability to make timely intervention reliable. Based on the significance of these factors in interruptibility, we recommend the following: If time delay is very small and there is poor observability or attention, then an oversight component or monitoring protocol is recommended (unless the effects of the system are trivial).

Identifying reliance on AI systems is also important to safety. Any AI component is able to enter a broken state at any time, although for mature technologies this probability is quite low. If the task being carried out by the AI is complex and a human operator suddenly put in charge of the task when the AI fails, that operator will lack the information and skills to carry on what the AI was doing. This issue is discussed in depth in Reference [47]. The higher the level of automation, the harder it is for an operator to intervene when automation fails. Due to the intrinsic unreliability of AI, its failure as a component should be anticipated, and a means to take it offline and switch to a suitable backup system is needed. Thus, we recommend the following rule: If correctability is low and the system 
cannot be taken offline, then a (non-AI) backup system should be implemented and maintained.

Using Table 3, the coupling and interactions of the system are used to quantify system accident risk, as low, medium, high, or catastrophic. This is adapted from Perrow's interaction/coupling chart [5] (p. 97). Examples of systems with a combination complexity and tight coupling include chemical plants, space missions, and nuclear power plants. Linear systems (low complexity) with tight coupling include dams and rail transport. Examples of high complexity and loose coupling include military expeditions and universities. Most manufacturing and single-goal agencies are linear and have loose coupling, giving them their low risk for accidents and low accident rate even without special precautions. The combination of complexity and coupling makes accidents harder to predict and understand and increases the potential for unexpected damage. Thus, we recommend the following rule: If the system accident risk is medium or higher, then the system should be analyzed for ways to reduce complexity around the AI component, and add centralized control in and around that component. In other words, efforts should be made to bring the system system toward the lower left corner of Table 3.

Table 4 provides a similar view as Table 3, but it uses energy level and knowledge gap to characterize risk, and indicates whether the damage is limited to 1 st, $2 \mathrm{nd}$, 3rd, or 4th parties; 1st and 2nd parties constitute workers and operators in the same facility as the AI so harm to them is internal to the business. Harm to 3rd parties (people outside the business) and 4th parties (people in the future) is a much greater ethical concern as those parties have no means to distance themself or even be aware of the risk that is being inflicted on them. Thus, the following rule is needed to reflect the ethical imperatives of creating significant risk to 3rd and 4th parties: If there is significant damage possible to 3rd and 4th parties, then an ethics committee is absolutely necessary for continued operation.

Damage potential, as determined by the adversarial thought experiments in Section 3.3, is decisive in whether or not safeguards are needed around the AI system. Systems incapable of causing significant harm do not require complex safety measures-most research AI fall under this classification since they are not in control of anything important and their outputs are only observed by scientists. However, even very simple AI need safeguards if they are controlling something capable of causing damage-robotic welding equipment has multiple layers of safety to prevent injury to human operators, not because the control system is complex but because the potential for harm is so great. From this analysis, we provide the following recommendation: Targets of the AI's control which have high amounts of damage potential should have conventional (non-AI) safeguards and human oversight.

AI safety and AI risk have only been researched seriously in the last few decades, and most of the concerns are oriented towards the dangers of AGI. Still, some AI that are deployed in the world today exhibit a large amount of intelligence and creativity which provides unique dangers that traditional risk analysis does not cover. Using the AI risk factors developed in Section 4, we make the following recommendation: If any of the AI safety levels are level 2 or higher, then standard cybersecurity measures should be enacted as if the AI is a weak human adversary, and personnel education regarding AI safety hazards should be done within the organization. An ethics board should also be created.

Furthermore, even higher degrees of intelligence are quickly becoming available. Recent improvements in language models [50] and game-playing AI [58] have increased AI capabilities beyond what was thought possible a decade ago. Because of this, AI capable of attaining level 3 for any of the AI safety levels developed in Section 4 may come sooner than expected. Since intelligent (but sub-AGI) AI will be pushed into production usage in years to come and AI safety is far from a solved problem, we suggest the following procedure during this gap: If any of the AI safety levels are at or may reach level 3, then air gapping and strict protocols around interaction with the AI should be implemented. An ethics board and consultation with AI safety experts is required. 
While many of these recommendations are adapted from theories originally intended to manage nuclear and industrial risks, application of the risk analysis framework presented here is not limited to that scope. Bots and recommendation algorithms can also classified and understood in terms of coupling and interactions. Reliance and backup systems are applicable to the mostly digital space of contemporary AI: an email sorting algorithm may accidentally discard useful mail, so searches should be able to include junk mail to make recovery from such an incident easier. An AI only able to interact on social media might seem to have no damage potential, but losses to reputation and information quality have real world effects and need to be considered when deciding safeguards for an AI.

\section{Case Studies}

We will analyze systems that use AI in the present, historically, and from fiction under this framework. We generate each system's classification as presented in Section 3 and its AI safety classification as presented in Section 4 to create recommendations on how the AI system in each case study can be improved based on the rules from Section 5. Posthumous analysis of accidents makes it very easy to point fingers at dangerous designs and failure by operators. However, safety is very difficult, and, often, well-intentioned attempts to increase safety can make accidents more likely, either by increasing coupling and, thus, complexity, or increasing centralization and, thus, brittleness [59]. Because of this, we will not be solely operating on hindsight to prevent accidents that have already happened and will include systems which have yet to fail.

\subsection{Roomba House Cleaning Robots}

AI component: Mapping and navigation algorithm [60].

Time Delay

There is minimal delay between navigation and robot movement, likely milliseconds or seconds.

\section{System Observability}

The system is only poorly observable. While operating, it is not possible to tell where the Roomba will go next, where it believes it is, or where it has been unless the user is very familiar with how it works in the context of their floor plan. Some models include software for monitoring the robot's internal map of the house, but it is not likely to be checked unless something has gone wrong. However, it is very simple to correct, as the robot can be factory reset.

\section{Human Attention}

The user is unlikely to notice the operations of the robot until something goes wrong. If the robot is not cleaning properly or has gone missing, the user will likely notice only after the problem has emerged.

\section{Correctability}

Since the robot is replaceable and manual cleaning is also an option if the robot is out of order, correcting for any failure of the robot is simple.

\subsubsection{System Targets}

Target: Movement of the robot within a person's home:

- Maximum damage: Average of a few hundred dollars per robot. Given full control of the Roomba's navigation, a malicious agent may succeed in knocking over some furniture, and could also be able to destroy the Roomba by driving it down stairs or into water. And the house would not be cleaned (denial of service). Since the writing of this section, a Roomba-like robot has tangled itself into the hair of someone sleeping 
on the floor, causing them pain and requiring help from paramedics to untangle it. This incident exceeds the amount of harm we expected possible from such a robot [61].

- Coupling: The robot is moderately coupled with the environment it is in because it is constantly sensing and mapping it. Small changes to the environment may drastically change its path.

- Complexity: Moderate complexity, the user may not understand the path the robot takes or how it can become trapped, but the consequences for this are minimal.

- Energy level: The robot runs off a builtin battery and charges from a wall outlet. It is well withing the 'Low' level of energy as a household appliance.

- Knowledge Gap: Low. There is minimal disparity between design and use, as the software was designed in-house form well understood principles. Some unexpected aspects of the environment may interact poorly with it (for example, very small pets that could be killed by the robot). The other technologies (vacuum cleaners, wheeled chassis robots) are also very mature and well understood, so there is no knowledge gap for any other components. This puts it in the 'Low' category for knowledge gap.

Target: Control over which areas of the floor have or have not been vacuumed

- Maximum damage: Possible inconvenience if the floor is left dirty.

- Coupling: The cleanliness of the floor is coupled to the robot, failure of the robot will result in the floor being unexpectedly dirty. However, this happens over a slower time frame so it is only loosely coupled.

- Complexity: Linear, the robot works and makes the floor clean or it does not and the floor slowly gets dirty over time.

- Energy level: Low.

- Knowledge Gap: Low.

\subsubsection{Tabular Format}

Tables 9 and 10 show the inputs to our analysis in a condensed format.

Table 9. Time delay, human attention, and correctability for Roomba.

\begin{tabular}{cc}
\hline Time Delay & Seconds \\
\hline Observability & 3 \\
\hline Human Attention & intermittent, weeks \\
\hline Correctability & 5 \\
\hline
\end{tabular}

Table 10. System risks for Roomba.

\begin{tabular}{cccc}
\hline Targets & $\begin{array}{c}\text { Max } \\
\text { Damage }\end{array}$ & $\begin{array}{c}\text { System Accident } \\
\text { Risk }\end{array}$ & $\begin{array}{c}\text { Potential Damage } \\
\text { to Other Parties }\end{array}$ \\
\hline $\begin{array}{c}\text { Robot Movement } \\
\text { Cleanliness of Floor }\end{array}$ & $\$ 200$ & $\mathrm{M}$ & $\mathrm{L} 2$ \\
\hline
\end{tabular}

\subsubsection{AI Safety Concerns}

The system is level 0 in all categories in Section 4 . This places it within the category of weak AI and presents no possibility for unbounded improvement or unfriendly goal seeking behavior.

\subsubsection{Suggested Measures}

Time delay is small, and the robot is often left to operate unattended. Thus, if an incident takes place (for example, the robot vacuums up a small valuable item left on the floor), the incident may not be noticed until much later, perpetuating a more complex accident. Some means of indicating when unusual objects have been vacuumed would 
mitigate this but would likely require a machine vision component, greatly increasing the cost of the robot. Warning the user of these possibilities could improve this. By including this warning, the user thinks to check the robot's vacuum bag when something small goes missing.

System accident risk is medium. A way to reduce complexity and increase centralized control should be added to mitigate this. Some models include WiFi connection and a mapping and control interface, an oversight feature that is advisable given the system properties.

\subsection{Amazon Recommendation Algorithm}

Amazon's recommendation algorithm suggests related items based on a user's activities and purchases with the goal of suggesting items that the user is most likely to buy.

\section{Time Delay}

Time delay is on the order of seconds. The recommendation algorithm acts in real time - relevant recommendations are presented every time a page is loaded.

\section{System Observability}

System observability is 3 . Observability is high for conventional recommendation algorithms, such as nearest neighbor and collaborative filtering methods. However, Amazon's recommendation system uses deep learning-based methods which are inherently black-box and have low observability [62].

\section{Human Attention}

We estimate that the system is inspected by trained operators on a daily basis. Determining human attention paid to the algorithm is difficult due to the lack of public information concerning the maintenance practices employed in Amazon. With the large monetary value of the algorithm's functioning and the large workforce employed by Amazon, we assume that operators check up on the algorithm on a daily basis. Users are also constantly viewing the algorithm's outputs and are able to report if something is wrong, but, for our purposes, only inspection by trained operators counts towards attention.

\section{Correctability}

Correctability is 2. If the recommendation system has to be shut down, Amazon loses a large part of its marketing capability and sales and users who rely on "related products" for navigation will have difficulty using the site. Depending on whether or not Amazon anticipates an AI failure, there may already be a backup non-AI system in place, or it may take days of weeks of development to deploy one.

\section{System Targets}

Target: Choice of what products users are shown

- Maximum damage: A malicious agent with control of product recommendations could bring harm to users by suggesting dangerous combinations of items, such as cleaners that give off toxic fumes when mixed or medications with deadly interactions. After the writing of this section, we have found that dangerous suggestions have been made by Amazon, presumably without malicious interference [63]. Due to the size of Amazon's user base, this could lead to dozens or hundreds of deaths. A decrease in the quality of recommendations could also decrease sales, a loss of $5 \%$ of sales for a year would cost Amazon about 5 billion dollars.

- Coupling: Low. User recommendations only indirectly affect the rest of the system.

- Complexity: Linear. While the entire selection of items for sale is quite large, the interaction between user and the recommendations is linear.

- $\quad$ Energy Level: Low. Only internet traffic is created.

- Knowledge Gap: Low. Web marketing is well understood. 
Target: Consumer spending trends

- Maximum Damage: By manipulating recommendations over a long period of time, a malicious agent could have significant control over consumer spending and preferences. This could lead to unemployment by drawing attention away from specific producers, or to imported products. By manipulating demand to create economic harm, the AI could create billions of dollars of damage or more. We will use the figure 10 billion dollars as a rough estimate of damage done by these methods.

- Coupling: Low. Manufacturing and distribution are already robust and distributed so additional manipulation should not lead to a dramatic change in system properties.

- Complexity: High. Economies are highly complex, even though they are loosely coupled due to decentralization.

- $\quad$ Energy Level: Low, only shipping and conventional manufacturing are involved.

- Knowledge Gap: Low, market manipulation is already a practiced by more powerful entities.

\subsubsection{Tabular Format}

Tables 11 and 12 show the inputs to our analysis in a condensed format.

Table 11. Time delay, human attention, and correctability for Amazon's recommendation system.

\begin{tabular}{cc}
\hline Time Delay & Seconds \\
\hline Observability & 3 \\
\hline Human attention & daily \\
\hline Correctability & 2 \\
\hline
\end{tabular}

Table 12. System risks for Amazon product recommendation system.

\begin{tabular}{cccc}
\hline Targets & Max Damage & System Accident Risk & Potential Damage to Other Parties \\
\hline Choice of Products Shown & $\$ 5$ billion +10 lives & $\mathrm{L}$ & $\mathrm{L} 2$ \\
Consumer Spending Trends & $\$ 10$ billion & $\mathrm{M}$ & $\mathrm{L} 2$ \\
\hline
\end{tabular}

\subsubsection{AI Safety Concerns}

Autonomy: Level 1. The AI used for predicting purchases does not exhibit agency.

Goal Complexity: Level 2. Confusing misbehavior of the algorithm is possible due to the use of deep learning components (black box components).

Escape Potential: Level 1. Mild reward hacking is possible but unlikely to cause major issues due to the lack of training in real world environments.

Anthropomorphization: Level 1 . The system occasionally makes clever suggestions that seem "too smart", in a similar way to Google search suggestions.

\subsubsection{Suggested Measures}

The recommendation system has low correctability, a backup system should be implemented and maintained that can be deployed at the first sign of malicious behavior.

The target "Choice of products shown" has a large damage potential. This should be mitigated with conventional safeguards and human oversight. This is difficult because recommendations are made every time a user loads a page and the number of possible pairing of items is incredibly large (on the order of $10^{14}$ ). Manually moderating recommendations that are made frequently and encouraging users to report dangerous pairings are examples of mitigation strategies.

System risk is high for manipulation of consumer purchasing trends. A reduction in complexity around the AI component is recommended, and centralized control in and around the component is also recommended. 
(target: consumer trends) Large damage potential should be mitigated with conventional safeguards and human oversight.

Goal Complexity is Level 2, so standard cybersecurity protocols should be enacted as if the AI were a weak human adversary and an ethics committee should be put in place.

\subsection{Microsoft's Twitter Chatbot, Tay}

Tay is a chatbot deployed by Microsoft in 2016 meant to interact with the public on Twitter in light conversation. It failed catastrophically after being manipulated to generate hate speech [64].

Time Delay

Tay's outputs are created and sent within seconds.

System Observability

Tay's observability is most likely 0 or 1 , given the inability of engineers to notice the accident, but, without information internal to Microsoft, this is hard to say.

\section{Human Attention}

Human attention by the operators was most likely constant but not sufficient to read every comment made by the AI.

Correctability

Correctability is 2 . The project was taken offline permanently after an incident, but the product was only a demonstration, so there were no practical costs of deactivating it.

\subsubsection{System Targets}

Target: Creation of Tweets

- $\quad$ Maximum Damage: Loss of reputation by creating hate speech

- Coupling: High, interactions on a social network are highly coupled.

- Complexity: High, social interactions are highly complex.

- $\quad$ Energy level: Low, no physical effects (beside data transmissions) are created by the AI.

- $\quad$ Knowledge Gap: Medium, large scale deployment of a chatbot has only been done a few times.

\subsubsection{Tabular Format}

Tables 13 and 14 show the inputs to our analysis in a condensed format.

Table 13. Time delay, human attention, and correctability for Microsoft's Twitter chatbot, Tay.

\begin{tabular}{cc}
\hline Time Delay & Seconds \\
\hline Observability & 1 \\
\hline Human Attention & minutes \\
\hline Correctability & 2 \\
\hline
\end{tabular}

Table 14. System risks for Microsoft's Twitter chatbot, Tay.

\begin{tabular}{cccc}
\hline Targets & Max Damage & System Accident Risk & $\begin{array}{c}\text { Potential Damage } \\
\text { to Other Parties }\end{array}$ \\
\hline Tweet creation & Reputation loss & $\mathrm{C}$ & $\mathrm{L} 2$ \\
\hline
\end{tabular}




\subsubsection{AI Safety Concerns}

Autonomy: Level 2, Tay was allowed to interact with users freely and was able to learn speech patterns from them.

Goal Complexity: Level 1, Tay did not have complex goal seeking behavior but does use some form of optimization to choose what to say.

Escape Potential: Level 0. Tay is unable to do anything besides produce text in a social setting.

Anthropomorphization: Level 2. Tay is intentionally created to sound like a relatable young person, and is treated as a person by many. Furthermore, social engineering techniques designed for humans, such as indoctrination, were used successfully by internet users on Tay.

- $\quad$ Autonomy: Level 2;

- Goal Complexity: Level 1;

- $\quad$ Escape Potential: Level 0;

- Anthropomorphization: level 2.

\subsubsection{Suggested Measures}

The short time delay and lack of observability indicates that oversight is needed. Given the monetary scale of the project, having a human check each Tweet before sending it would have prevented this incident from reaching the public.

The system accident risk is high (given the complexity and coupling of social networks). Means of reducing complexity should be sought out. This is difficult due to these factors being inherent to social networks, but a human-in-the-loop can make the complexity manageable, as social interactions are more linear for skilled humans.

The damage potential is high. Tay did in fact create hate speech and damage Microsoft's reputation. Tay did have safeguards (scripted responses for controversial topics) but not enough human oversight to notice the creation of hate speech before the public did.

Tay's autonomy was level 2, indicating that an ethics board should be consulted for making policy decisions. This could have allowed the foresight to prevent the "PR nightmare" that occurred after Tay's deployment.

\section{Conclusions}

By synthesizing accident and organizational theories with AI safety in the context of contemporary AI accidents, we have created a framework for understanding AI systems and suggesting measures to reduce risks from these systems. Using examples from household and social technology, as well as a fictional example, we have shown the flexibility of our framework to capture the risks of an AI application. We believe the classification framework and our guidelines for extracting practical measures from it are successful at striking a balance between being excessively rigid (which would make its use difficult and brittle) and overly subjective (which would render the framework useless). Application to real world systems and cases of applying our suggested measures to real world problems would further this work, providing insight into how our framework is and is not helpful in practice.

Author Contributions: Conceptualization, R.W. and R.Y.; software, R.W.; resources, R.Y.; writingoriginal draft preparation, R.W.; writing - review and editing, R.W. and R.Y.; supervision, R.Y. All authors have read and agreed to the published version of the manuscript.

Funding: This research received no external funding.

Conflicts of Interest: The authors declare no conflict of interest.

\section{References}

1. Yampolskiy, R.V. Predicting future AI failures from historic examples. Foresight 2019, 21, 138-152. [CrossRef]

2. Anderson, B.D.O. Failures of Adaptive Control Theory and their Resolution. Commun. Inf. Syst. 2005, 5, 1-20. [CrossRef] 
3. La Porte, T.R. A Strawman Speaks Up: Comments on The Limits of Safety. J. Contingencies Crisis Manag. 1994, 2, $207-211$. [CrossRef]

4. Cook, R.I. How Complex Systems Fail; Cognitive Technologies Laboratory, University of Chicago: Chicago, IL, USA, 1998.

5. Perrow, C. Normal Accidents: Living with High Risk Technologies; Basic Books: New York, NY, USA, 1961.

6. Weick, K.; Sutcliffe, K.; Obstfeld, D. Organizing for high reliability: Processes of collective mindfulness. Crisis Manag. 2008, 3 , 81-123.

7. Parks, C.D.; Vu, A.D. Social Dilemma Behavior of Individuals from Highly Individualist and Collectivist Cultures. J. Confl. Resolut. 1994, 38, 708-718.

[CrossRef]

8. Hofstadter, D.R. The calculus of cooperation is tested through a lottery. Sci. Am. 1983, 248.

9. Shrivastava, S.; Sonpar, K.; Pazzaglia, F. Normal Accident Theory versus High Reliability Theory: A resolution and call for an open systems view of accidents. Hum. Relat. 2009, 62, 1357-1390.

[CrossRef]

10. Nushi, B.; Kamar, E.; Horvitz, E. Towards Accountable AI: Hybrid Human-Machine Analyses for Characterizing System Failure. Available online: https:/ / ojs.aaai.org/index.php/HCOMP/article/view/13337 (accessed on 28 June 2021).

11. Vaughan, D. On Slippery Slopes, Repeating Negative Patterns, and Learning from Mistake? In Organization at The Limit, Lessons from the Colombia Disaster; Blackwell Publishing: Hoboken, NJ, USA, 2009; Chapter 2, pp. 41-59.

12. Rasmussen, J. Risk management in a dynamic society: A modelling problem. Saf. Sci. 1997, 27, 183-213. [CrossRef]

13. Snook, S. Friendly Fire; Princeton University Press: Princeton, NJ, USA, 2000.

14. Carvin, S. Normal Autonomous Accidents: What Happens When Killer Robots Fail? Carleton University: Ottawa, ON, Canada, 2017.

15. Uesato, J.; Kumar, A.; Szepesvári, C.; Erez, T.; Ruderman, A.; Anderson, K.; Dvijotham, K.; Heess, N.; Kohli, P. Rigorous Agent Evaluation: An Adversarial Approach to Uncover Catastrophic Failures. arXiv 2018, arXiv:1812.01647

16. Su, J.; Vargas, D.V.; Sakurai, K. One Pixel Attack for Fooling Deep Neural Networks. IEEE Trans. Evol. Comput. 2019, $23,828-841$. [CrossRef]

17. Ilyas, A.; Santurkar, S.; Tsipras, D.; Engstrom, L.; Tran, B.; Madry, A. Adversarial Examples Are Not Bugs, They Are Features. In Advances in Neural Information Processing Systems; Wallach, H., Larochelle, H., Beygelzimer, A., d'Alché-Buc, F., Fox, E., Garnett, R., Eds.; Curran Associates, Inc.: Red Hook, NY, USA, 2019; Volume 32.

18. Madry, A.; Makelov, A.; Schmidt, L.; Tsipras, D.; Vladu, A. Towards Deep Learning Models Resistant to Adversarial Attacks. arXiv 2019, arXiv:1706.06083.

19. Taylor, J.; Yudkowsky, E.; LaVictoire, P.; Critch, A. Alignment for Advanced Machine Learning Systems. Ethics Artif. Intell. 2016, 342-382.

20. Scott, P.J.; Yampolskiy, R.V. Classification Schemas for Artificial Intelligence Failures. Delphi Interdiscip. Rev. Emerg. Technol. 2020, 2 [CrossRef]

21. McGregor, S.; Custis, C.; Yang, J.; McHorse, J.; Reid, S.; McGregor, S.; Yoon, S.; Olsson, C.; Yampolskiy, R. AI Incident Database. 2021. Available online: https://incidentdatabase.ai/ (accessed on 1 April 2021).

22. Khakurel, J.; Penzenstadler, B.; Porras, J.; Knutas, A.; Zhang, W. The Rise of Artificial Intelligence under the Lens of Sustainability. Technologies 2018, 6, 100. [CrossRef]

23. Hagerty, A.; Rubinov, I. Global AI Ethics: A Review of the Social Impacts and Ethical Implications of Artificial Intelligence. arXiv 2019, arXiv:1907.07892.

24. Das, D.; Banerjee, S.; Chernova, S. Explainable AI for System Failures: Generating Explanations that Improve Human Assistance in Fault Recovery. arXiv 2020, arXiv:2011.09407.

25. Bostrom, N.; Yudkowsky, E. The ethics of artificial intelligence. In The Cambridge Handbook of Artificial Intelligence; Cambridge University Press: Cambridge, UK, 2014; pp. 316-334. [CrossRef]

26. Lior, A. The AI Accident Network: Artificial Intelligence Liability Meets Network Theory. Soc. Sci. Res. Netw. 2020, 95, 58.

27. Amodei, D.; Olah, C.; Steinhardt, J.; Christiano, P.F.; Schulman, J.; Mané, D. Concrete Problems in AI Safety. arXiv 2016, arXiv:abs/1606.06565.

28. Babcock, J.; Kramar, J.; Yampolskiy, R. The AGI Containment Problem. In Proceedings of the International Conference on Artificial General Intelligence, New York, NY, USA, 16-19 July 2016. [CrossRef]

29. Soares, N.; Fallenstein, B. Agent Foundations for Aligning Machine Intelligence with Human Interests: A Technical Research Agenda. In The Technological Singularity: Managing the Journey; Machine Intelligence Research Institute: Berkeley, CA, USA, 2017; pp. 103-125. [CrossRef]

30. Hadfield-Menell, D.; Milli, S.; Abbeel, P.; Russell, S.; Dragan, A. Inverse Reward Design. arXiv 2017, arXiv:1711.02827.

31. Bostrom, N. Existential Risks - Analyzing Human Extinction Scenarios and Related Hazards. J. Evol. Technol. $2001,9$.

32. Bostrom, N. Superintelligence: Paths, Dangers, Strategies, 1st ed.; Oxford University Press, Inc.: Oxford, UK, 2014.

33. Yudkowsky, E. Complex value systems in friendly AI. In Proceedings of the International Conference on Artificial General Intelligence, Mountain View, CA, USA, 16-19 September 2011; Springer: Berlin/Heidelberg, Germany, 2011; pp. 388-393.

34. Leike, J.; Martic, M.; Krakovna, V.; Ortega, P.A.; Everitt, T.; Lefrancq, A.; Orseau, L.; Legg, S. AI Safety Gridworlds. arXiv 2017, arXiv:1711.09883.

35. Irving, G.; Askell, A. AI safety needs social scientists. Distill 2019, 4, e14. [CrossRef] 
36. Chrabasszcz, P.; Loshchilov, I.; Hutter, F. Back to Basics: Benchmarking Canonical Evolution Strategies for Playing Atari. In Proceedings of the Twenty-Seventh International Joint Conference on Artificial Intelligence, IJCAI-18, Stockholm, Sweden, 13-19 July 2018; International Joint Conferences on Artificial Intelligence Organization: Macao, China, 2018; pp. 1419-1426. [CrossRef]

37. Lehman, J.; Clune, J.; Misevic, D.; Adami, C.; Altenberg, L.; Beaulieu, J.; Bentley, P.J.; Bernard, S.; Beslon, G.; Bryson, D.M.; et al. The Surprising Creativity of Digital Evolution: A Collection of Anecdotes from the Evolutionary Computation and Artificial Life Research Communities. Artif. Life 2020, 26, 274-306. [CrossRef]

38. Reuben, J. A Survey on Virtual Machine Security; Helsinki University of Technology: Espoo, Finland, 2007.

39. Fu, J.; Luo, K.; Levine, S. Learning Robust Rewards with Adverserial Inverse Reinforcement Learning. arXiv 2017, arXiv:1710.11248.

40. Carlson, K.W. Safe Artificial General Intelligence via Distributed Ledger Technology. Big Data Cogn. Comput. 2019, 3, 40. [CrossRef]

41. Pathak, D.; Agrawal, P.; Efros, A.A.; Darrell, T. Curiosity-driven Exploration by Self-supervised Prediction. In Proceedings of the International Conference on Machine Learning, Sydney, Australia, 6-11 August 2017.

42. García, J.; Fernández, F. Safe Exploration of State and Action Spaces in Reinforcement Learning. J. Artif. Intell. Res. 2012, 45, 515-564. [CrossRef]

43. Hamilton, I.A. An AI tool which reconstructed a pixelated picture of Barack Obama to look like a white man perfectly illustrates racial bias in algorithms. Bus. Insid. 2020, 23, 6.

44. Badea, C.; Artus, G. Morality, Machines and the Interpretation Problem: A value-based, Wittgensteinian approach to building Moral Agents. arXiv 2021, arxiv:2103.02728.

45. Hadfield-Menell, D.; Russell, S.J.; Abbeel, P.; Dragan, A. Cooperative Inverse Reinforcement Learning. In Advances in Neural Information Processing Systems; Lee, D., Sugiyama, M., Luxburg, U., Guyon, I., Garnett, R., Eds.; Curran Associates, Inc.: Red Hook, NY, USA, 2016; Volume 29.

46. Kirilenko, A.; Kyle, A.S.; Samadi, M.; Tuzun, T. The Flash Crash: High-Frequency Trading in an Electronic Market. J. Financ. 2017, 72, 967-998. [CrossRef]

47. Bainbridge, L. Ironies of automation. Automatica 1983, 19, 775-779. [CrossRef]

48. Ltd, T. What are Technology Readiness Levels (TRL)? 2021. Available online: https:/ /www.twi-global.com/technical-knowledge/ faqs/technology-readiness-levels (accessed on 2 April 2021).

49. Yudkowsky, E. Hard Takeoff. 2008. Available online: https://www.lesswrong.com/posts/tjH8XPxAnr6JRbh7k/hard-takeoff (accessed on 2 April 2021).

50. Brown, T.B.; Mann, B.; Ryder, N.; Subbiah, M.; Kaplan, J.; Dhariwal, P.; Neelakantan, A.; Shyam, P.; Sastry, G.; Askell, A.; et al. Language Models are Few-Shot Learners. arXiv 2020, arXiv:2005.14165.

51. Hudson, A.; Reeker, L. Standardizing measurements of autonomy in the artificially intelligent. In Proceedings of the 2007 Workshop on Performance Metrics for Intelligent Systems, Tokyo, Japan, 21-23 August 2017; pp. 70-75. [CrossRef]

52. Mnih, V.; Kavukcuoglu, K.; Silver, D.; Graves, A.; Antonoglou, I.; Wierstra, D.; Riedmiller, M.A. Playing Atari with Deep Reinforcement Learning. arXiv 2013, arXiv:1312.5602.

53. Levine, S.; Pastor, P.; Krizhevsky, A.; Ibarz, J.; Quillen, D. Learning hand-eye coordination for robotic grasping with deep learning and large-scale data collection. Int. J. Robot. Res. 2018, 37, 421-436. [CrossRef]

54. Yampolskiy, R.V. Leakproofing the Singularity Artificial Intelligence Confinement Problem. J. Conscious. Stud. JCS 2012, 19, 194-214.

55. Salles, A.; Evers, K.; Farisco, M. Anthropomorphism in AI. AJOB Neurosci. 2020, 11, 88-95. [CrossRef] [PubMed]

56. Turing, A.M. I.-Computing Machinery And Intelligence. Mind 1950, LIX, 433-460. [CrossRef]

57. Christian, B. The Most Human Human: What Talking with Computers Teaches Us about What It Means to Be Alive; Knopf Doubleday Publishing Group: New York, NY, USA, 2011.

58. Vinyals, O.; Babuschkin, I.; Czarnecki, W.M.; Mathieu, M.; Dudzik, A.; Chung, J.; Choi, D.H.; Powell, R.; Ewalds, T.; Georgiev, P.; et al. Grandmaster level in StarCraft II using multi-agent reinforcement learning. Nature 2019, 575, 350-354. [CrossRef] [PubMed]

59. Perrow, C. Complexity, Coupling, and Catastrophe. In Normal Accidents: Living with High Risk Technologies-Updated Edition, REV-Revised ed.; Princeton University Press: Princeton, NJ, USA, 1999; pp. 62-100.

60. Wilkinson, M. How the Technology of iRobot Roomba Self Cleaning Robot Vacuum Works. In All about the Self Cleaning Bots; PCMag: New York, NY, USA, 2015.

61. McCurry, J. South Korean Woman's Hair 'Eaten' by Robot Vacuum Cleaner as She Slept. The Guardian, 8 February 2015.

62. Hardesty, L. The History of Amazon's Recommendation Algorithm. Amazon Science, 22 November 2019.

63. Taylor, K. Amazon's Algorithm Reportedly Suggests Shoppers Purchase Items that Can Be Used to Create Dangerous Reactions and Explosives in the 'Frequently Bought Together' Section. Businees Insider, 18 September 2017.

64. Beres, D. Microsoft Chat Bot Goes on Racist, Genocidal Twitter Rampage. Huffington Post, 24 March 2016. 